\title{
ADICIONES A LA AVIFAUNA DEL PARQUE NACIONAL MBURUCU- YA, CORRIENTES, ARGENTINA.
}

\author{
P. D. CANO(1); C. SAibene(2); M. L. ChatellenaZ(3); H. A. BAll(2) y A. Vallejos(2)
}

RESUMEN: Aportamos nueva información sobre la composición, hábitat y distribución de la avifauna del Parque Nacional Mburucuyá. Presentamos datos de diez especies cuya presencia en el Parque no había sido registrada con anterioridad. Además mencionamos otras cinco especies que, aunque contaban con registros previos, poseen particular interés para esta área protegida por su valor para la conservación. El presente trabajo acrecienta el conocimiento de la avifauna del Parque y representa una importante herramienta para la planificación del manejo en el área protegida.

ABSTRACT: We provide new information on the composition, habitat and distribution of birds from the Mburucuyá National Park. We present data of ten species whose presence in the park had not been registered previously. Also mention other five species with previous records by its value for conservation. This work increases the knowledge of the birds in the park and is an important tool for management planning in the protected area.

Palabras Claves: aves; especies amenazadas; conservación; áreas protegidas.

Key Words: birds, endangered species, conservation, protected areas.

\section{INTRODUCCIÓN}

El conocimiento de las áreas naturales y de las especies de interés para la conservación que en ellas habitan, es una fuente de información primaria para la generación de estrategias de investigación, manejo y conservación. La Provincia de Corrientes, posee 16 AICAs (Areas importantes para la conservación de las aves en Argentina), las cuales albergan 20 especies globalmente amenazadas y tres especies endémicas de biomas (Di Giacomo, 2005). Una de estas áreas es el Parque Nacional Mburucuyá, es el único Parque Nacional del Sistema Nacional de Areas Protegidas existente en la provincia de Corrientes. El primer inventario de especies de aves del Parque fue realizado por J. Hutton entre 1980 y 1990 (Saibene, 2005). Posteriormente el mismo fue completado con aportes de Gómez y Babarskas (1992), aunque ese inventario permaneció como un informe interno de la Administración de Parques Nacionales. Chebez et al. (1998) basados en estos registros, citaron un total de 293 especies en el Parque Nacional Mburucuyá, 84 de las cuales fueron consideradas nidificantes. De esta manera, el Parque Nacional Mburucuyá se ubicó entre los cinco parques nacionales de Argentina con mayor riqueza de aves. No obstante, en ambos informes varias especies fueron señaladas como pendientes de confirmación o como de presencia dudosa.

(1) Centro de Zoología Aplicada. Universidad Nacional de Córdoba. daniganges@yahoo.com.ar

(2) Administración de Parques Nacionales.

(3) Facultad de Ciencias Exactas y Naturales y Agrimensura, UNNE. 
Con el objetivo de ampliar el conocimiento respecto a la distribución de las especies y aportar nueva información sobre la composición de su avifauna, presentamos aquí datos de diez especies cuya presencia en el Parque Nacional Mburucuyá no había sido registrada con anterioridad. Además mencionamos otras cinco especies que, aunque contaban con registros previos, poseen particular interés para ésta área protegida por su valor para la conservación.

\section{AREA DE ESTUDIO}

El Parque Nacional Mburucuyá posee una superficie de 17.680 ha y está ubicado en el noroeste de la provincia de Corrientes, en el departamento Mburucuyá (57 $59^{\circ}$ y $58^{\circ} 08^{\prime} \mathrm{W} ; 2^{\circ} 58^{\prime}$ y $28^{\circ} 05^{\prime} \mathrm{S}$ ) (Fig. 1). El área pertenece al Sistema de Esteros del Iberá, incluidos en la Cuenca del Plata, uno de los humedales más importantes de Latinoamérica (Canevari et al., 1998). El sector sur del Parque está limitado por el Estero Santa Lucía, que ocupa alrededor de 4000 ha del área protegida. El sector norte del Parque está ocupado por las cañadas Portillo y Fragosa, las que suman aproximadamente 3700 ha. Ambas cañadas desaguan en el estero Santa Lucía a través del Arroyo Portillo. Además, existen numerosas lagunas ubicadas sobre las lomadas arenosas (Saibene y Montanelli, 1997).

Según Cabrera (1976), el área está incluida en el Distrito Oriental de la Provincia Fitogeográfica Chaqueña. Aunque, también existen especies vegetales características de la Provincia Paranaense y del Espinal. La vegetación se compone básicamente de pajonales, pastizales e isletas de bosque higrófilo y sub-xerófilo. Superpuestos a las comunidades herbáceas, existen palmares de Butia yatay (Saibene y Montanelli, 1997). Hacia las zonas bajas, en sectores con drenaje deficiente, crecen formaciones vegetales hidrófilas, como praderas de ciperáceas. Los esteros están bordeados por una franja ocupada por Cyperus giganteus, Scirpus californicus, Typha domingensis, Thalia spp., entre otras (Saibene y Montanelli, 1997).

El clima se caracteriza por tener poca amplitud térmica a lo largo del año, la temperatura media anual es de $21,5^{\circ} \mathrm{C}$ y los promedios de temperatura media anual oscilan entre $21^{\circ} \mathrm{C}$ y $23^{\circ} \mathrm{C}$ (Montanelli y González, 1998). Las precipitaciones, originadas por los vientos húmedos que ingresan desde el Atlántico, alcanzan medias anuales superiores a $1200 \mathrm{~mm}$ (Montanelli y González, 1998). El régimen es de carácter estacional, la época más lluviosa es el otoño y la más seca es el invierno (Stamatti, 2002).

\section{RESULTADOS}

Registramos la presencia de diez especies no citadas previamente: Anas platalea, Harpyhaliaetus coronatus, Tringa flavipes, Calidris melanotos, Larus cirrocephalus, Sittasomus griseicapillus, Tityra inquisitor, Cistothorus platensis, Anthus chacoensis y Anthus furcatus. Además, confirmamos la presencia de Caprimulgus longirostris, citada como "de presencia dudosa" (Chebez et al., 1998). Estas adiciones elevan a 308 especies 
el inventario de la avifauna del Parque Nacional Mburucuyá. Además, brindamos información sobre otras cinco especies, que si bien ya contaban con registros previos, merecieron a nuestro juicio comentarios adicionales. Éstas fueron: Xolmis dominicanus, Alectrurus risora, Emberizoides ypiranganus, Ictinia mississippiensis y Trogon surrucura.

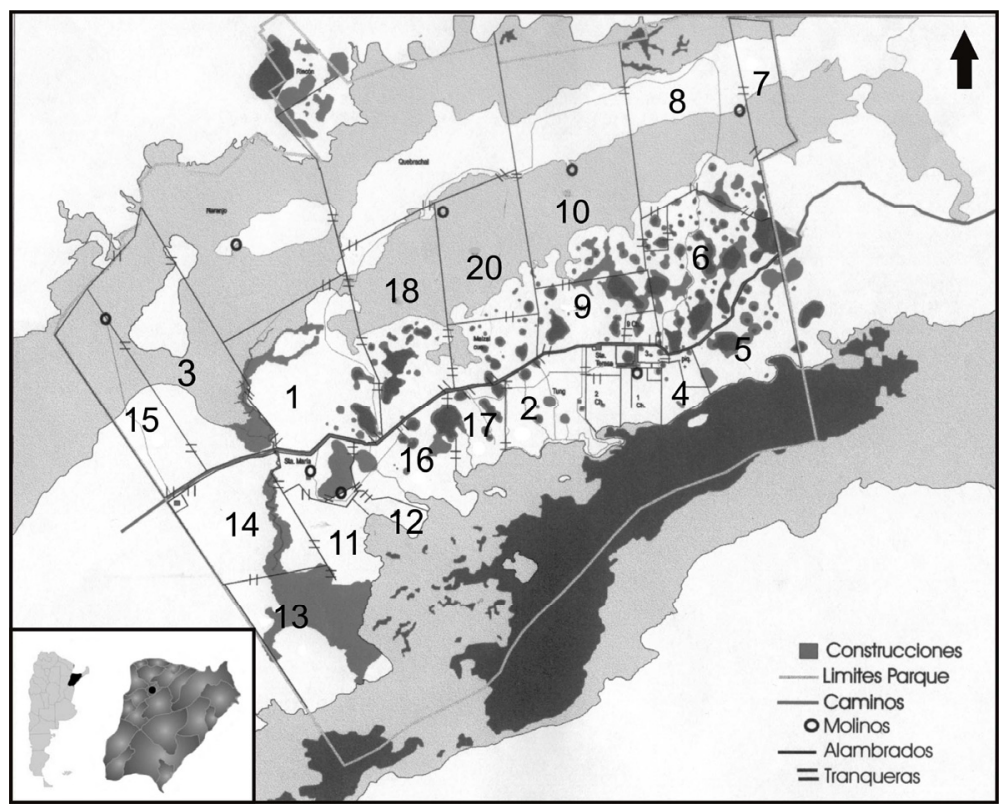

Fig. 1: Mapa del Parque Nacional Mburucuyá. Se destaca la ubicación y límites de los potreros mencionados en el texto.

\section{Comentarios sobre nuevas especies registradas}

\section{Pato Cuchara (Anas platalea)}

El 13 de junio de 2009 observamos y fotografiamos 7 individuos alimentándose en una laguna situada en el límite este del Parque (Potrero 5). Otro grupo de 8 individuos pasó en vuelo a corta distancia. Una hora después, registramos un individuo nadando y alimentándose en la laguna ubicada en el Casco Histórico Santa Teresa. Esta especie no contaba con registros previos para esta área protegida (Gómez y Babarskas, 1992; Chebez et al., 1998; Hutton y Schimff, 2001). Consideramos que esta especie es ocasional en el Parque.

\section{Aguila Coronada (Harpyhaliaetus coronatus)}

Para esta especie, Amenazada a nivel mundial (BirdLife International, 2010) y En Peligro en Argentina (López-Lanús et al., 2008), se estima una población de menos de 1000 individuos (Maceda, 2007). Su distribución en Argentina abarca desde el noroeste, nor- 
deste y centro, hasta la provincia de Río Negro y en el sur de Buenos Aires (De la Peña y Rumboll, 1998; Narosky e Yzurieta, 2003; Rodríguez Mata et al., 2006). A diferencia de otras grandes águilas neotropicales, esta especie se distribuye principalmente en bosques y estepas arbustivas xerófilas en zonas bajas y en menor proporción habita sabanas y bosques húmedos subtropicales y tropicales, tanto de llanura como montanos (Gonnet y Blendinger 1998). Uno de los primeros requerimientos tendientes a la conservación de la especie debe ser identificar áreas con poblaciones de tamaño razonable para la supervivencia de la especie (Collar et al. 1992). El 10 de agosto de 1996 Judith Hutton registró esta especie en la ruta Provincial $\mathrm{N}^{\circ} 6$ entre las localidades de Mburucuyá y Ramones (com. pers.), pero hasta ahora no se contaba con registros dentro del área protegida. Registramos esta especie en el Parque en dos oportunidades: la primera, en octubre de 2003, un individuo en el potrero Quebrachal. El 18 de diciembre 2006, observamos 2 individuos posados en un eucalipto a una altura aproximada de $15 \mathrm{~m}$, en cercanía de la Seccional Santa María.

\section{Pitotoy Chico (Tringa flavipes)}

El 20 de febrero de 2006 observamos y fotografiamos 2 ejemplares de Tringa flavipes caminado sobre la orilla arenosa de una laguna (potrero 17). El 26 de febrero de 2006, en el Estero Santa Lucía, observamos y fotografiamos un ejemplar de esta especie caminando sobre un sustrato de lodo y vegetación acuática. Dos especies del mismo género $T$. melanoleuca y $T$. solitaria, han sido mencionadas con anterioridad para el Parque Nacional Mburucuyá (Gómez et al., 1992).

\section{Playerito Pectoral (Calidris melanotos)}

El 15 de febrero de 2006, durante la realización del Censo Neotropical de Aves Acuáticas, observamos un ejemplar de Calidris melanotos en una playa con arena a orillas el Arroyo Portillo. El 16 de febrero de 2006 fotografiamos un ejemplar caminando en la orilla arenosa de una laguna (potrero 17). Nuevamente, el día 20 de febrero de 2006 avistamos un individuo caminando en la orilla de una laguna con abundante vegetación (potrero 16). Esta especie, de amplia distribución en Argentina (Canevari et al., 1991) no había sido registrada previamente en el Parque.

\section{Gaviota Capucho Gris (Larus cirrocephalus)}

En julio de 2006 sobre el Estero Santa Lucía observamos 8 ejemplares de Larus cirrocephalus en vuelo. Es una especie es Migrante Austral Parcial, pasa el invierno en territorio argentino donde frecuenta espejos de agua dulce, costas de ríos y lagunas, de paso por su ruta migratoria por el Este de Sudamérica (Canevari et al., 1991; Narosky e Yzurieta, 2003). Consideramos que esta especie es ocasional en el Parque.

\section{Tarefero (Sittasomus griseicapillus)}

En febrero de 2004 observamos un ejemplar en el estrato arbóreo de una isleta de bosque higrófilo (potrero Tung). Aunque el área de distribución de este dendrocoláptido abarca 
gran parte de la provincia de Corrientes (Olrog 1979, Contreras 1987) y se la citó para isletas de bosques del noroeste de Corrientes (Chatellenaz 2004), no había sido registrada previamente en el Parque.

\section{Tueré Chico (Tityra inquisitor)}

El 21 de febrero de 2004 observamos un ejemplar macho de esta especie posado a más de $10 \mathrm{~m}$ de altura, en un eucalipto en el acceso al Casco Histórico Santa Teresa. Este es el primer registro de la especie para el Parque.

\section{Ratona Aperdizada (Cistothorus platensis)}

Registramos esta especie en dos oportunidades: el 23 de julio de 2005 en el potrero 17 y el 15 de mayo de 2006 en el potrero 5, en el límite este del Parque. En ambos casos se la observó posada en hierbas sobresalientes en pajonales de Andropogon lateralis de más de un metro de altura.

\section{Cachirla Trinadora (Anthus chacoensis)}

Esta especie es mencionada para Corrientes por Contreras (1987). Stotz et al. (1996) sugirieron que merece una alta prioridad de conservación y estudio. Collar et al. (1992) mencionaron la especie para la localidad San Cayetano (Corrientes), en base a colectas de J. Contreras. También es mencionada para la provincia de Corrientes por De la Peña (1999) y Casañas et al. (2007). Esta especie se distingue de sus congéneres por diferencias en su canto y un particular despliegue nupcial (Chebez, 1994). Observamos 2 ejemplares el 17 de enero de 2005 en el borde de la Laguna Enfrentada Sur (potrero 17), bordeada por una franja de pastizales bajos. Observamos otros 2 ejemplares el 26 de julio de 2004 en el borde de la Laguna Preferida (potrero 2 Grande). Constituyen los primeros registros de esta especie para el Parque.

\section{Cachirla Uña Corta (Anthus furcatus)}

Mencionada para Corrientes por Contreras (1987), aunque no estaba citada para el Parque. Observamos un ejemplar el 5 de mayo de 2004 y otros 2 ejemplares el día 26 de julio de 2004 en el borde de la Laguna Enfrentada Sur (potrero 17). Estos constituyen los primeros registros de esta especie para el Parque.

\section{Atajacaminos Nañarca (Caprimulgus longirostris)}

Esta especie de amplia distribución en Argentina, fue indicada como de presencia dudosa por Chebez et al. (1998). Sin embargo la observamos en el Parque entre los años 2004 y 2009 en diferentes oportunidades, posada sobre la ruta provincial $\mathrm{N}^{\circ} 86$. 


\title{
Comentarios sobre otras especies de importancia
}

\author{
Monjita Dominicana (Xolmis dominicanus)
}

Esta especie es considerada Vulnerable a nivel mundial (BirdLife International, 2010b) y En Peligro en Argentina (López-Lanús et al., 2008). Además, es considerada Especie de Valor especial (EVE) para el Parque (Administración de Parques Nacionales, 2002). Su hábitat está siendo modificado rápidamente por cambios en el uso de la tierra. En esta zona particularmente por forestaciones de árboles no nativos de Eucalyptus y Pinus spp. donde se ha llegado a alterar radicalmente los remanentes de pastizales, especialmente en Entre Ríos y Corrientes (Pearman y Abadie, 1995; Fraga 2003). Registramos esta especie con frecuencia fuera del Parque en el lugar conocido como "Sedario", zona donde la Cañada Fragosa es atravesada por la ruta Provincial No 86 y cuenta con pocos registros en el Parque. El 16 de mayo de 2006 observamos 2 machos y 2 hembras posados en el alambrado que constituye el límite oeste del parque (potrero 15). La vegetación de este sitio está compuesta por pajonales de Andropogon lateralis, atravesados por una intrincada red de canalículos encharcados ("malezales", en el sentido de Carnevali (1994)). Durante la mayor parte del período de observación (30 minutos), realizaron frecuentes vuelos hacia un potrero de la estancia vecina al Parque, donde se hallaban aproximadamente 30 vacas, y el pasto era muy corto $(5-10 \mathrm{~cm})$. Posaban en el suelo, entre el ganado, y sobre termiteros desde donde realizaban cortos vuelos para capturar insectos. El 15 de marzo de 2007 observamos 3 ejemplares en zonas de pastizales húmedos y malezales (potrero 3). El 16 de mayo del mismo año, observamos 7 ejemplares, en las orillas de la Cañada Fragosa al norte del Parque (potrero Rincón).

\section{Yetapá de Collar (Alectrurus risora)}

Esta especie es considerada Vulnerable a nivel mundial (BirdLife International, 2010a) y En Peligro en Argentina (López-Lanús et al., 2008). Además, es considerada Especie de Valor Especial (EVE) para el Parque (Administración de Parques Nacionales, 2002). Habita pastizales y pajonales húmedos en esteros o bañados, algunas veces cerca de los bordes de bosques, vegetación arbustiva y sabanas con palmares (Short, 1975; Olrog, 1979; Canevari et al., 1991). Las principales amenazas mencionadas en Argentina para esta especie son la pérdida y destrucción del hábitat debido a la agricultura y sobrepastoreo en pastizales naturales. Además, la modificación del hábitat por la forestación con Eucalyptus y Pinus spp. (Di Giacomo y Krapovickas, 2001; Coconier y Di Giacomo, 2009). En Corrientes y Formosa, la ganadería no ha producido modificaciones críticas de los pastizales naturales (Di Giacomo y Krapovickas, 2001; Di Giacomo y Di Giacomo 2004). Sin embargo, en Corrientes las quemas de primavera y verano para promover las pasturas podrían afectar los sucesos reproductivos (Chebez et al., 1998; Azpiroz in litt., 1999). No obstante se ha observado la construcción de nidos, cercanos al suelo o sobre matas en rebrotes, en pastizales post-quemas (Di Giacomo y Di Giacomo 2004). Aunque en el Parque esta especie es poco frecuente, la registramos tanto en períodos reproductivos como no reproductivos. En el Parque habita los pastizales altos principalmente de la 
zona norte del área protegida, donde en ocasiones hemos podido observar y fotografiar individuos machos reproductivos, no reproductivos, hembras y juveniles (Fig. 2).

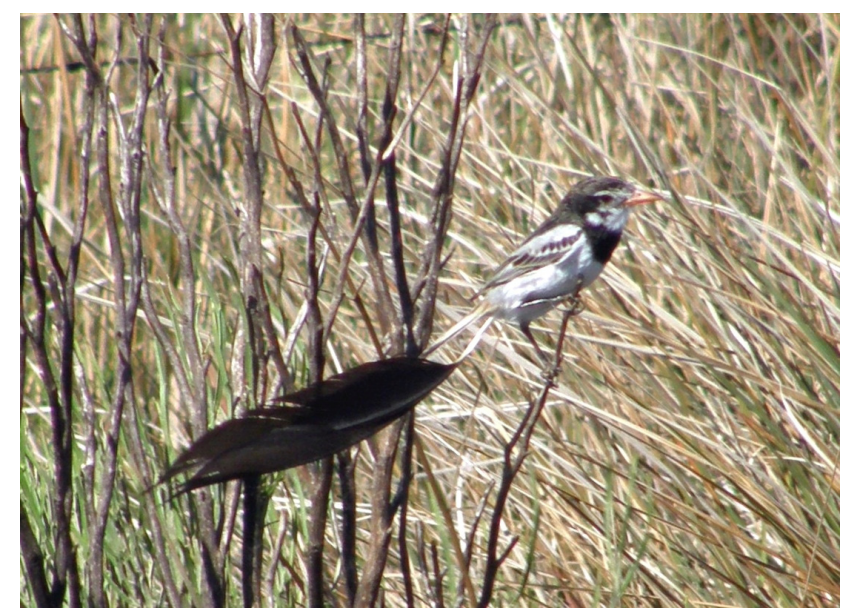

Fig. 2: Individuo macho de Alectrurus risora en plumaje de reposo sexual en el Parque Nacional Mburucuyá, agosto de 2008. Foto: Héctor Ball.

\section{Coludo Chico (Emberizoides ypiranganus)}

Esta especie que habita bañados y pastizales (Tobias et al., 1997) se encuentra categorizada como Especie Vulnerable en Argentina por López-Lanús et al. (2008). Dentro del Sistema Nacional de Areas Protegidas solamente se encuentra en el Parque Nacional Mburucuyá, donde es relativamente común y está considerada Especie de Valor Especial (EVE) para este Parque (Administración de Parques Nacionales, 2002). Se la encuentra en pajonales húmedos, de más de 1 metro de altura, de Andropogon lateralis y Sorghastrum aff. agrostoides. En el Parque la registramos en varias oportunidades en el borde del Estero Santa Lucía. Esta especie se encuentra incorrectamente descripta e ilustrada en algunas de las guías de campo disponibles, como resultado puede ser pasada por alto, sin embargo es menos rara de lo que generalmente se piensa (Tobias et al., 1997).

\section{Milano Boreal (Ictinia mississippiensis)}

Esta especie Migrante Neártica está presente en Argentina en primavera y verano. Aunque ya había sido registrada en el Parque (Gómez et al., 1992), desde el año 2004 fue observada en varias oportunidades en grandes grupos de hasta aproximadamente 200 ejemplares volando en forma circular y a bajas alturas, y además posados en los árboles más altos de las isletas de bosque higrófilo.

\section{Surucuá Común (Trogon surrucura)}

Esta especie típica de selva y bosques, aunque citada previamente por Chebez et al. (1998) y Hutton y Schimff (2001) había sido observada en el Parque en pocas ocasiones. 
En enero de 2000 registramos visual y auditivamente esta especie en dos ocasiones en el Casco Histórico Santa Teresa. En febrero del año 2005 avistamos y fotografiamos un ejemplar posado en un árbol en cercanías del Centro de Informes del Parque. Durante los años 2008 y 2009 esta especie fue registrada en tres oportunidades en isletas de bosque higrófilo, de aproximadamente 500 ha (Cano y Nores, 2009) desde entonces cuenta con diversos registros en el parque.

\section{CONCLUSIÓN}

El presente trabajo brinda nueva información sobre la composición, hábitat y distribución de la avifauna del Parque Nacional Mburucuyá, esto permite contar con una base para abordar estudios focalizados en especies particulares. Además, representa una importante herramienta para la planificación del manejo de esta área protegida. Consideramos prioritario el abordaje de estudios poblacionales y sobre la biología de las especies aquí mencionadas, principalmente aquellas que se encuentran en alguna categoría de amenaza. A su vez, recomendamos el seguimiento de sus poblaciones a lo largo del tiempo incluyendo en los estudios a aquellas variables que podrían afectar la permanencia y conservación de las especies en el área, particularmente aquellas relacionadas con el manejo de los pastizales y pajonales del parque.

\section{AgRAdECIMIENTOS}

Agradecemos al Dr. J.M. Chani, quien con sabiduría y experiencia estimuló la elaboración del proyecto marco de esta publicación. Al Guardaparque Nacional A. Carmarán y a Judith Hutton por la valiosa información brindada del Aguila Coronada. A nuestros asistentes de campo: N. Acevedo, M. Barrios, M. Castelino, E. Planas I Figueras, M. Del Huerto, A. Fernández, Y. Lezcano, M. Andreau, E. Alderete y M. Olmedo Masat. A la Delegación Técnica NEA de la Administración de Parques Nacionales que nos facilitó material bibliográfico y renovaciones de los permisos de investigación. Además, agradecemos a los revisores del manuscrito.

\section{BIBLIOGRAFÍA}

Administración de Parques Nacionales, 2002. Plan General de Manejo del Parque Nacional Mburucuyá. Iguazú, Argentina. 186 p.

BIRDLIFE INTERNATIONAL, 2009. Red List of Threatened Species. Version 2010.1. http://www.iucnredlist.org on $19 / 10 / 2010$.

BIRDLIFE INTERNATIONAL, 2010a. Species factsheet: Alectrurus risora. Downloaded from http://www.birdlife.org on 19/10/2010.

BirdLife International, 2010b. Species factsheet: Xolmis dominicanus. Downloaded from http://www.birdlife.org on 19/10/2010. 
CABrerA, A.L., 1976. Regiones fitogeográficas argentinas. Enciclopedia Argentina de Agricultura y Jardinería II. $1^{\circ}$ y $2^{\circ}$ Edición. ACME. Buenos Aires, Argentina. 85 p.

CAnevari, M.; P. CAnevari; G.R. CARrizo; G. Harris; J. RodríGuez Mata y R.J. Straneck, 1991. Nueva Guía de las Aves Argentinas. Tomo II. Fundación Acindar. Buenos Aires, Argentina. 497 p.

Canevari, P.; D. Blanco; E.H. Bucher; G. Castro y I. Davidson, 1998. Los Humedales de Argentina: Clasificación, Situación Actual, Conservación y Legislación. Wetlands International. Publicación, 46, Buenos Aires, Argentina. 48 p.

CANO, P.D. y M. NoRes, 2009. Avifauna de isletas naturales de selva (mogotes) en nordeste argentino en relación a la Teoría de Biogeografía de Islas. Página 119. Libro de Resúmenes, Reunión Argentina de Ornitología, Tafí del Valle, Tucumán, Argentina.

Carnevali, R., 1994. Fitogeografía de la Provincia de Corrientes. Ediciones del autor, Corrientes, Argentina. 324 pp.

CASAÑAs, H.E.; I. Roesler y J. KLAvins, 2007. Historia Natural y distribución de la Cachirla Trinadora (Anthus chachoensis). Hornero, 22: 59-63. Buenos Aires, Argentina.

Chatellenaz, M.L., 2004. La avifauna del bosque de Quebracho Colorado y Urunday del noroeste de Corrientes, Argentina. Facena, 20: 3-12. Corrientes, Argentina.

Chebez, J.C., 1994. Los que se van. Especies argentinas en peligro. Editorial Albatros. Buenos Aires, Argentina. $604 \mathrm{p}$.

Chebez, J.C.; N. Rey; M. Barbaskas y A.D. Giacomo, 1998. Las aves de los Parques Nacionales de la Argentina. Administración de Parques Nacionales y Asociación Ornitológica del Plata. Monografía Especial. Editorial L.O.L.A. N 12. Buenos Aires, Argentina. 127 p.

Coconier, E.G. y A.S. Di Giacomo, 2009. Argentina. Pp. 59-70. En: C. Devenish; D.F. Díaz Fernández; R.P. Clay; I. Davidson and I. Yépez Zabala (Eds): Important Bird Areas Americas Priority sites for biodiversity conservation. Quito, Ecuador: BirdLife International (BirdLife Conservation Series $\mathrm{N}^{\circ} 16$ ).

Collar N.J.; L.P. Gonzaga; N. Krabbe; A. Madroño Nieto; N.G. NARAnjo; T.A. III Parker and D.C. WEGE, 1992. Threatened birds of the Americas. The ICBP/IUCN Red Data Book. International Council for Bird Preservation, Cambridge, UK.

CONTRERAS, J.R., 1987. Lista preliminar de la avifauna correntina. II. Passeriformes. Historia Natural. Volumen 7, $\mathrm{N}^{\circ}$ 6, páginas 61-70. Corrientes, Argentina.

De LA PeÑA, M.R., 1999. Aves argentinas, lista y distribución. Monografía Especial LOLA N¹8. Buenos Aires, Argentina.

De la Peña, M.R. and M. Rumboll, 1998. Birds of southern South America and Antarctica. Harper Collins, Londres.

Di Giacomo, A.S. (Ed.), 2005. Areas de Importancia para la Conservación de las Aves en Argentina. Sitios prioritarios para la conservación de la biodiversidad. Temas de naturaleza y Conservación 5. Aves Argentinas/Asociación Ornitológica del Plata. Buenos Aires, Argentina. 514 p.

Di Giacomo, A.S. and S.K. KrapovicKAS, 2001. Afforestation threatens Argentina's grasslands. World Bird Watch, 23: 24-25.

Di GiAcOmo, A.S. and A.G. Di GiACOMO, 2004. Extinción, historia natural y conservación de las poblaciones del Yetapá de Collar (Alectrurus risora) en la Argentina. Ornitología Neotropical, 15 Suppl.:145157.

FRAGA, R., 2003. Distribution, natural history and conservation of de Black-and-white Monjita (Heteroxolmis dominicana) in Argentina, a species vulnerable to extinction. Ornitología Neotropical, 14: 157-171. 
GonNet, J.M. y P.G. Blendinger. 1998. Nuevos registros de distribución del Aguila coronada (Harpyhaliaetus coronatus) en el oeste de Argentina. Hornero, 15: 39-42.

Gómez, D. y M. Babarskas, 1992. Aves. Pp. 27-43. En: Gómez, D.; A. Bosso; S. Heinonen; A. Giraudo; M. Babarskas; J. Baldo y G. Marino (eds.): La naturaleza de las estancias Santa María y Santa Teresa, Mburucuyá, provincia de Corrientes. Proyecto Parque Nacional Mburucuyá. Informe inédito. Administración de Parques Nacionales. Buenos Aires, Argentina.

Hutton, J. y S. SchimfF, 2001. Parque Nacional Mburucuyá. Lista de Aves. Folleto. Administración de Parques Nacionales, Buenos Aires. Argentina.

LóPez-Lanús, B.; P. Grilli; E. Coconier; A. Di Giacomo y R. BAnchS, 2008. Categorización de las aves de la Argentina según su estado de conservación. Informe de Aves Argentinas/AOP y Secretaría de Ambiente y Desarrollo Sustentable. Buenos Aires, Argentina. 64 p.

MACEDA, J.J., 2007. Biología y conservación del Águila Coronada (Harpyhaliaetus coronatus) en Argentina. Hornero, 22 (2):159-171. Buenos Aires. Argentina.

Montanelli, S. y L. GonZÁleZ, 1998. Caracterización climática del Parque Nacional Mburucuyá. Informe Inédito, Administración de Parques Nacionales. Corrientes, Argentina.

Narosky, T. y D. Yzurieta, 2003. Guía para la identificación de la Aves de Argentina y Uruguay. Edición de Oro. Vázquez Mazzini Editores. Buenos Aires, Argentina.

Olrog, C.C., 1979. Nueva lista de la avifauna Argentina. Opera Lilloana, 27:1-324. Tucumán, Argentina.

PEARMAn, M. and E.I. ABADIE, 1995. Mesopotamia grasslands and wetlands survey, 1991-1993: conservation of threatened birds and habitat in northeast Argentina. Unpublished report.

Rodríguez Mata, J.; F. ERIZE y M. Rumboll, 2006. Aves de Sudamérica. No paseriformes. Desde ñandúes a carpinteros. Letemendia Casa Editora. Buenos Aires, Argentina.

SAIBENE, C., 2005. Parque Nacional Mburucuyá. Pp. 152-153. En: A.S. Di Giacomo (Ed.): Areas de Importancia para la Conservación de las Aves en Argentina. Sitios prioritarios para la conservación de la biodiversidad. Temas de naturaleza y Conservación 5. Aves Argentinas/AOP, Buenos Aires, Argentina.

Saibene C. y S. Montanelli, 1997. Mapeo de las comunidades leñosas del Parque Nacional Mburucuyá, Corrientes, Argentina. Facena, 13: 49-57.

ShORT, L.L., 1975. A zoogeographic analysis of the South American Chaco avifauna. Bulletin of American Museum Natural History, 154: 163-352.

Stamatтi, G., 2002. Propuesta de zonificación del Parque Nacional Mburucuyá, Corrientes, Argentina. Tesis de Maestría, Centro de Zoología Aplicada, Universidad Nacional de Córdoba, Córdoba, Argentina.

Stotz D.F.; J.W. FitzPATRICK; T.A. III PARKER and D.F. MoskoviTs, 1996. Neotropical birds. Ecology and Conservation. University of Chicago Press, Chicago.

TOBIAS, J.A.; R.P ClAY and J.C. LowEN, 1997. Field identification of Lesser Grass-finch Emberizoides ypiranganus. Cotinga, 8: 75-78. 